\title{
Growth patterns and body composition in former extremely low birth weight (ELBW) neonates until adulthood: a systematic review
}

\author{
Caroline Van de Pol $^{1}$ (D) $\cdot$ Karel Allegaert ${ }^{2,3}$ \\ Received: 25 June 2019 /Revised: 10 December 2019 / Accepted: 12 December 2019 \\ (C) Springer-Verlag GmbH Germany, part of Springer Nature 2020
}

\begin{abstract}
Preterm infants are obviously born lighter and shorter, with smaller head circumferences than normal birth weight term born neonates. They also have a different body composition. Compromised growth is associated with adverse health outcomes. Both growth retardation and accelerated growth are suggested to cause metabolic, cardiovascular, and renal complications. Reviews regarding growth and body composition in preterm infants often do not differentiate between birth weight and gestational age. The purpose of this systematic review is to assemble growth data, specific in extremely low birth weight children. Different databases were searched for studies regarding growth and body composition in former extremely low birth weight infants until adulthood. We compared height, weight, head circumference, body mass index, fat mass, lean mass, fat distribution, and body water to matched normal birth weight controls and the World Health Organization growth charts. Studies consistently reported that former extremely low birth weight neonates experience a period of accelerated postnatal growth, but they achieve lower anthropometric parameters than normal birth weight children. There is no consensus about differences in body composition and how to measure this.

Conclusion: Although extremely low birth weight infants exhibit a period of catch-up growth, their growth remains retarded later in life. Further research is needed to investigate body composition and the associated risk of cardiovascular diseases or metabolic syndrome.
\end{abstract}

What is Known:

- Extremely low birth weight infants have lower anthropometric parameters and a different body composition at birth and term-corrected age than normal birth weight infants.

- Former extremely low birth weight infants also have a higher risk on adverse cardiovascular health outcomes in later life.

What is New:

- After hospital discharge, extremely low birth weight neonates remain smaller and probably also lighter, with smaller head circumferences at each corrected age throughout childhood and adolescence when compared to normal birth weight infants or the World Health Organization growth charts. It is not clear whether extremely low birth weight infants reach a lower or similar body mass index score as normal birth weight infants.

- There is a lack of (long-term) information on body composition in extremely low birth weight infants.

Keywords Anthropometry $\cdot$ Body composition $\cdot$ Extremely low birth weight $\cdot$ Fat distribution $\cdot$ Growth $\cdot$ Total body fat

Karel Allegaert

karel.allegaert@uzleuven.be

Caroline Van de Pol

caroline.vandepol@gmail.com

1 KU Leuven, Leuven, Belgium

2 Department of Development and Regeneration, and Department of Pharmaceutical and Pharmacological Sciences Organ Systems, KU Leuven, Leuven, Belgium

3 Department of Clinical Pharmacy, Erasmus MC, Erasmus MC, Rotterdam, The Netherlands

$\begin{array}{ll}\text { Abbreviations } \\ \text { AGA } & \text { Appropriate for gestational age } \\ \text { BMI } & \text { Body mass index } \\ \text { BW } & \text { Birth weight } \\ \text { CA } & \text { Corrected age } \\ \text { CPEG } & \text { Canadian Pediatric Endocrine Group } \\ \text { ELBW } & \text { Extremely low birth weight } \\ \text { FM } & \text { Fat mass } \\ \text { FMI } & \text { Fat mass index } \\ \text { \%FM } & \text { Percentage fat mass } \\ \text { GA } & \text { Gestational age } \\ \text { HC } & \text { Head circumference }\end{array}$

\section{Abbreviations}

BMI Body mass index

BW Birth weight

CA Corrected age
CPEG - Canadian Pediatric Endocrine Group
ELBW Extremely low birth weight

Fat mass

$\%$ FM Percentage fat mass

HC Head circumference 


$\begin{array}{ll}\text { LM } & \text { Lean mass } \\ \text { LMI } & \text { Lean mass index } \\ \text { \%LM } & \text { Percentage lean mass } \\ \text { NBW } & \text { Normal birth weight } \\ \text { TBF } & \text { Total body fat } \\ \text { \%TBF } & \text { Percentage total body fat } \\ \text { TBW } & \text { Total body water } \\ \text { \%TBW } & \text { Percentage total body water } \\ \text { SGA } & \text { Small for gestational age } \\ \text { TCA } & \text { Term corrected age } \\ \text { WHO } & \text { World Health Organization } \\ \text { SSE } & \text { Sum of squared estimate of errors } \\ \text { RMSE } & \text { Root mean square error }\end{array}$

\section{Introduction}

Growth patterns and body development are relevant in preterm infants, likely most relevant in extremely low birth weight (ELBW, i.e., $<1 \mathrm{~kg}$ ) infants. Infants born preterm are lighter and shorter, with a smaller head circumference (HC) than their term born normal birth weight (NBW) peers, at birth as well as when they reach term-corrected age (TCA) and throughout childhood $[1,2]$. When preterm infants are classified by weight, as ELBW infants, they are often small for their gestational age (SGA) [3]. This makes them even more susceptible to effects of perinatal growth retardation [4].

The perinatal growth retardation is followed by a period of catch-up growth, which usually starts in early infancy and can continue into early adolescence [5].

Preterm infants do not only have aberrant growth measurements but they also have a different body composition compared to term born neonates. Because the third trimester is a period of rapid adipose tissue deposition, they exhibit a fat distribution at TCA different than that of term born neonates. Preterm infants have a lower fat mass (FM) and an even more decreased lean mass (LM) at TCA, resulting in a greater percentage total body fat (\%TBF) than term born infants [2].

Low birth weight (LBW) infants, and even more ELBW infants are more susceptible to coronary heart diseases, neonatal acute kidney injury, chronic kidney disease, type 2 diabetes, stroke, and hypertension [6-8].

Although the effects of (early) infancy weight gain on later body composition and health outcomes are not clear, associations with an aberrant fat distribution, cardiovascular diseases, and an obesity-associated exacerbation of renal risk have been suggested [8-12]. It is suggested that an altered body composition that persists into adulthood could be a risk factor for developing metabolic and cardiovascular diseases, although it could not always be identified as a causal factor [3, 5, 9, 13, 14]. However, the ideal growth pattern for ELBW infants is not yet known.
In addition, analyzing body composition could be useful for calculating drug doses and body composition parameters are used in some prediction models to determine maintenance doses [15].

Literature reviews regarding body composition after hospital discharge and later in life are scarce and most do not differentiate between gestational age (GA) or birth weight (BW), although they might be important predictors of body composition [16].

This systematic search focuses specifically on ELBW infants and investigates their growth patterns and body composition after hospital discharge throughout infancy and adulthood. Using a systematic review approach, we compared height, weight, $\mathrm{HC}$, and body mass index (BMI) to control data of NBW peers and growth charts described by the World Health Organization (WHO) and the Canadian Pediatric Endocrine Group (CPEG). Parameters regarding body fat and fat distribution were compared to control data in the included studies.

\section{Materials and methods}

\section{Study characteristics}

\section{Study design}

We performed a systematic search and review including both prospective and retrospective longitudinal studies, as well as cross-sectional studies that describe data in cohorts of former ELBW neonates after hospital discharge until adulthood. There was no limit on minimum or maximum follow-up time. We only included observational studies. Intervention studies were excluded.

\section{Language restrictions}

Besides studies and abstracts that were available in English, studies written in a different language were included if they had an abstract or schematics available in English that contained the desired data.

\section{Patients}

We included studies concerning former ELBW infants. ELBW is defined as a BW less than $1000 \mathrm{~g}$. ELBW infants are either SGA or appropriate for their GA (AGA). SGA is defined as a BW less than the 10th percentile for weight given the GA [17-22], but studies are heterogeneous as they sometimes use different terms and definitions to indicate SGA. Intra-uterine growth restriction is also used as an analogous definition. 
For control data, we simultaneously collected the data of control NBW (i.e., 2500-4000 g) infants matched by sex and age in the concerned study whenever available, and we compared the growth parameters to the WHO and CPEG growth charts [23-25].

\section{Search strategy and selection process}

A first search was performed by the first author in July 2018 and a second one in December 2018. Databases PubMed, Cochrane Library, Web of Science, and Embase were searched.

The extended search strategies for different databases and selection of the articles are represented in Table 1 and Fig. 1, respectively [26]. The quality of the English studies was assessed using the "Quality Assessment Tool for Observational Cohort and Cross-Sectional Studies" of the National Heart, Lung and Blood Institute [27]. Studies were selected based on title and abstract, and the second author was consulted in case of uncertainties.

\section{Outcome parameters and methodology applied}

Parameters that were a component of growth or body composition were extracted by the first author. In case of uncertainties, the second author was consulted. Extracted growth parameters are height, weight, HC, and BMI. Parameters regarding body composition were either absolute (i.e., FM, LM, and total body water $(\mathrm{TBW}))$ or relative $(\% \mathrm{TBF}$, percentage lean mass (\%LM), and percentage total body water (\% TBW)). We also investigated fat distribution.

\section{Growth}

We compared mean height, weight, $\mathrm{HC}$, and $\mathrm{BMI}$ with data of NBW control children when available in the studies and with the 5th, 25th, and 50th percentile of the standard growth parameters as described in the WHO Child Growth Standards and in the WHO Reference 2007 [23-25]. For weight-for-age after the age of 10, we compared with extended WHO reference curves provided by the CPEG. Since these charts do not provide data for the 5 th percentile, we compared to the 3 th percentile instead [28].

We plotted the calculated weighted mean data of boys and girls against the WHO growth charts. The WHO data were calculated as the weighted mean of boys and girls equal to the ratio of male to female patients in the concerned studies at the compared age. We plotted growth data in graphs edited in MATLAB, while using a rational function based on the entered data [29]. Individual function characteristics of the graphs are described below the figures. The WHO growth charts are used to describe trends among the growth of
ELBW infants, but they were not statistically compared with the study data.

Different definitions of catch-up growth are used in the included studies. Catch-up growth is defined as a positive increase of the Z-score of $>0.67$ over a certain period of time $[20,30]$ or reaching growth parameters more than -2 SD scores for age [22, 31].

\section{Body composition}

For FM, LM, \% TBF, and \%LM, we compared mean data of ELBW infants to control data as described in the included studies.

\section{Methods to measure growth parameters and body composition}

Studies were not excluded based on methods used for measuring growth parameters or body composition, as some of these methods are suggested to be more accurate but also more expensive or less applicable to newborn infants [14]. The use of these methodologies is further explained in the studies.

\section{Results}

There were 486 search results in PubMed, 33 trials in Cochrane Library, 383 in Embase, and 192 in Web of Science. Two studies that met the selection criteria were excluded to avoid duplication (the study populations were also included in another more comprehensive included study) [32, 33]. Eventually, a total of 16 studies and one abstract were included. The selection process of the articles is represented in the PRISMA flow chart in Fig. 1 [26]. An overview of the included studies and the quality assessment are represented in Table 2 and Table 3, respectively. One study had a very low follow-up rate with a small remaining study sample and was therefore not used for the WHO comparison [38]. Results of growth compared to controls are represented in Table 4 and those of body composition data in Table 5 .

\section{Growth}

\section{Height}

In almost every included study that compared ELBW infants to NBW infants, children and adults born with an ELBW remain smaller than NBW children, at every stage of their development [17-21, 30, 35-37, 39-41]. Two studies found a difference between genders with a lower height in male infants at the age of 11 [41] and in female infants at the age of 14.7 [20]. Two studies suggested that ELBW infants attain 
Table 1 Search strategies for different databases

\section{Database Search strategy}

Pubmed ( ) ("Extremely Low Birth Weight Infant"[Mesh] OR "Extremely low birth weight infant*”[tiab] OR "ELBW”[tiab] OR "Extremely Low Birth Weight"[tiab])) AND ("Body Constitution"[Mesh/NoExp] OR "Body Constitution*"[tiab] OR "Body Distribution"[tiab] OR "Body weights and measures"[Mesh/NoExp] OR "body weights and measures"[tiab] OR "Body Measure*”[tiab] OR “morphometry"[tiab] OR "Morphometrics"[tiab] OR "Body Fat Distribution"[Mesh] OR "Body Fat Distribution"[tiab] OR "Body Fat Patterning”[tiab] OR "Adiposity”[tiab] OR "Adipose tissue distribution"[tiab] OR "Fat tissue distribution"[tiab] OR "Fatty Tissue Distribution"[tiab] OR "Body Mass Index"[Mesh] OR "Body Mass Index"[tiab] OR "Quetelet* Index"[tiab] OR "BMI”[tiab] OR "Body Size"[Mesh/NoExp] OR "Body Size*”[tiab] OR "Body Mass"[tiab] OR "Body Height"[Mesh/NoExp] OR "Body Height*"[tiab] OR "Body length"[tiab] OR"Body Weight"[Mesh/NoExp] OR "Body Weight*"[tiab] OR "Total Body Weight"[tiab] OR "Body Weight Changes"[Mesh/NoExp] OR "Body Weight Change*”[tiab] OR "Body Composition”[Mesh] OR "Body Composition*"[tiab] OR "Body Water"[mesh] OR "body water"[tiab] OR "Whole Body Water"[tiab] OR "Total Water"'[tiab] OR "Total Body Water"[tiab] OR "Total Body Fluid"[tiab] OR "adipose tissue"[Mesh/NoExp] OR “Adipose Tissue*”[tiab] OR "Fat Tissue"[tiab] OR "Fatty Tissue"[tiab] OR "Fat Pad"”[tiab] OR "Body Fat"[tiab] OR "body lipid"[tiab] OR "visceral fat distribution"[tiab] OR "Subcutaneous Fat distribution"[tiab] OR “Anthropometry"[Mesh/NoExp] OR “Anthropometry”[tiab] OR “Anthropometric index"[tiab] OR “Anthropometrics”[tiab] OR "Anthropometric parameters"[tiab] OR "Cephalometry"[mesh] OR "Cephalometry"[tiab] OR "Cephalometrics"[tiab] OR "Craniometry"[tiab] OR “Craniometrics"[tiab] OR "Head circumference"[tiab] OR "Cranial Circumference"[tiab] OR "Fat Mass"[tiab] OR "Fat Free Mass"[tiab] OR "Lean Mass"[tiab] OR ("Postdischarge"[tiab] or "Postdischarge"[ tiab] AND ("growth”[mesh] OR "growth"[tiab])))

Cochrane \#1[mh "Extremely Low Birth Weight Infant"]

Library \#2("Extremely low Birth Weight Infant" or "ELBW" or "Extemely low birth weight"):ti,ab,kw

\section{\#3\#1 OR \#2}

\#4[mh ^"Body Constitution"] or [mh^"Body Weights and measures"] or [mh "Body Fat Distribution"] or [mh "Body Mass Index"] or [mh ^"Body Size"] or [mh ^"Body Height"] or [mh ^"Body Weight"] or [mh ^"Body Weight Changes"] OR [mh "Body Composition"] or [mh "Body Water"] or [mh $\wedge$ "Adipose tissue"] or [mh^"Anthropometry"] or [mh "Cephalometry"]

\#5("Body Constitution" or "Body Distribution" or "body weights and measures" or "Body Measure*" or "morphometry" or "Morphometrics" or "Body Fat Distribution" or "Body Fat Patterning" or "Adiposity" or "Adipose tissue distribution" or "Fat tissue distribution" or "Fatty Tissue Distribution" or "Body Mass Index" or "Quetelet* Index" or "BMI" or "Body Size*” or "Body Mass" or "Body Height*” or "Body length" or "Body Weight*" or "Total Body Weight" or "Body Weight Change*" or "Body Composition*" or "body water" or "Whole Body Water" or "Total Water" or "Total Body Water" or "Total Body Fluid" or "Adipose Tissue*" or "Fat Tissue" or "Fatty Tissue" or "Fat Pad*" or "Body Fat" or "body lipid" or "visceral fat distribution" or "Subcutaneous Fat distribution" or "Anthropometry" or "Anthropometric index" or "Anthropometrics" or "Anthropometric parameters" or "Cephalometry" or "Cephalometrics" or "Craniometry" or "Craniometrics" or "Head circumference" or "Cranial Circumference" or "Fat Mass" or "Fat Free Mass" or "Lean Mass"):ti,ab,kw

\#6("Postdischarge" or "Post-Discharge"):ti,ab,kw

$\# 7[\mathrm{mh} \wedge$ "Growth"]

\#8(“Growth"):ti,ab,kw

$\# 9 \# 7$ OR \#8

$\# 10 \# 6$ and \#9

$\# 11 \# 5$ OR \#10

\#12\#4 OR \#11

\#13\#3 AND \#12

EMBASE ('extremely low birth weight'/exp. OR 'extremely low birth weight':ti,ab OR 'elbw':ti,ab OR 'extremely low birth weight infant*':ti,ab) AND ('body constitution'/exp. OR 'body constitution':ti,ab OR 'body distribution':ti,ab OR 'morphometry'/mj OR 'morphometry':ti,ab OR 'morphometrics':ti,ab OR 'body weights and measures':ti,ab OR 'body measure*':ti,ab OR ‘body composition'/exp. OR 'body composition':ti,ab OR 'body fat':ti,ab OR ‘body lipid':ti,ab OR ‘fat load':ti,ab OR ‘body fat distribution':ti,ab OR ‘body fat patterning':ti,ab OR 'adipose tissue distribution':ti,ab OR 'fat tissue distribution':ti,ab OR 'fatty tissue distribution':ti,ab OR 'subcutaneous fat distribution':ti,ab OR 'visceral fat distribution':ti,ab OR 'body water':ti,ab OR 'total water':ti,ab OR 'whole body water':ti,ab OR 'total body water':ti,ab OR 'total body fluid':ti,ab OR 'adipose tissue'/mj OR 'adipose tissue':ti,ab OR 'fat tissue':ti,ab OR 'fatty tissue':ti,ab OR ‘fat pad'/exp. OR 'fat pad*':ti,ab OR 'anthropometry'/mj OR 'anthropometry':ti,ab OR 'anthropometric index':ti,ab OR 'anthropometrics':ti,ab OR 'antropometry':ti,ab OR 'body measurement':ti,ab OR 'cephalometry'/mj OR 'cephalometry':ti,ab OR 'cephalometrics':ti,ab OR 'head size':ti,ab OR 'craniometry'/mj OR 'craniometry':ti,ab OR 'craniometrics':ti,ab OR 'anthropometric parameters'/mj OR 'anthropometric parameters':ti,ab OR 'body height'/exp. OR 'body height':ti,ab OR 'body length':ti,ab OR 'body weight'/mj OR 'body weight':ti,ab OR 'total body weight':ti,ab OR 'body weight change*':ti,ab OR 'body mass'/exp. OR 'body mass':ti,ab OR 'bmi':ti,ab OR 'body mass index':ti,ab OR 'quetelet* index':ti,ab OR 'body size'/exp. OR 'body size':ti,ab OR 'fat free mass'/exp. OR 'fat free mass':ti,ab OR 'fat mass'/exp. OR 'fat mass':ti,ab OR 'lean mass':ti,ab OR 'head circumference'/exp. OR 'head circumference':ti,ab OR 'cranial circumference':ti,ab OR (('postdischarge':ti,ab OR 'post-discharge':ti,ab) AND ('growth'/mj OR 'growth':ti,ab)))

Web of Science TS = (("Extremely low birth weight infant*” OR "ELBW" OR "Extremely Low Birth Weight") AND ("Body Constitution*” OR "Body Distribution" OR "body weights and measures" OR "Body Measure*” OR "morphometry" OR "Morphometrics" OR "Body Fat Distribution" OR "Body Fat Patterning" OR “Adiposity" OR "Adipose tissue distribution" OR "Fat tissue distribution" OR "Fatty Tissue Distribution" OR "Body Mass Index" OR "Quetelet* Index" OR "BMI" OR "Body Size*” OR "Body Mass" OR "Body Height*” OR "Body length" OR "Body Weight*” OR "Total Body Weight" OR "Body Weight Change*" OR "Body Composition*" OR "body water" OR "Whole Body Water" OR "Total Water" OR "Total Body Water" OR "Total Body Fluid" OR "Adipose Tissue*" OR "Fat Tissue" OR "Fatty Tissue" OR "Fat Pad*” OR "Body Fat" OR "body lipid" OR "visceral fat distribution" OR "Subcutaneous Fat distribution" OR "Anthropometry" OR "Anthropometric index" OR "Anthropometrics" OR "Anthropometric parameters" OR "Cephalometry" OR "Cephalometrics" OR "Craniometry" OR "Craniometrics" OR "Head circumference" OR "Cranial Circumference" OR "Fat Mass" OR "Fat Free Mass" OR "Lean Mass" OR ((“Postdischarge" OR "Post-discharge") AND "growth”)))

$E L B W$ Extremely low birth weight, $B M I$ Body mass index, Ti title, $A b$ abstract, $M h$ MeSH, NoExp unexploded, Tiab title or abstract, $M j$ major focus, Exp explode, $T S$ top 
Fig. 1 PRISMA 2009 flow diagram

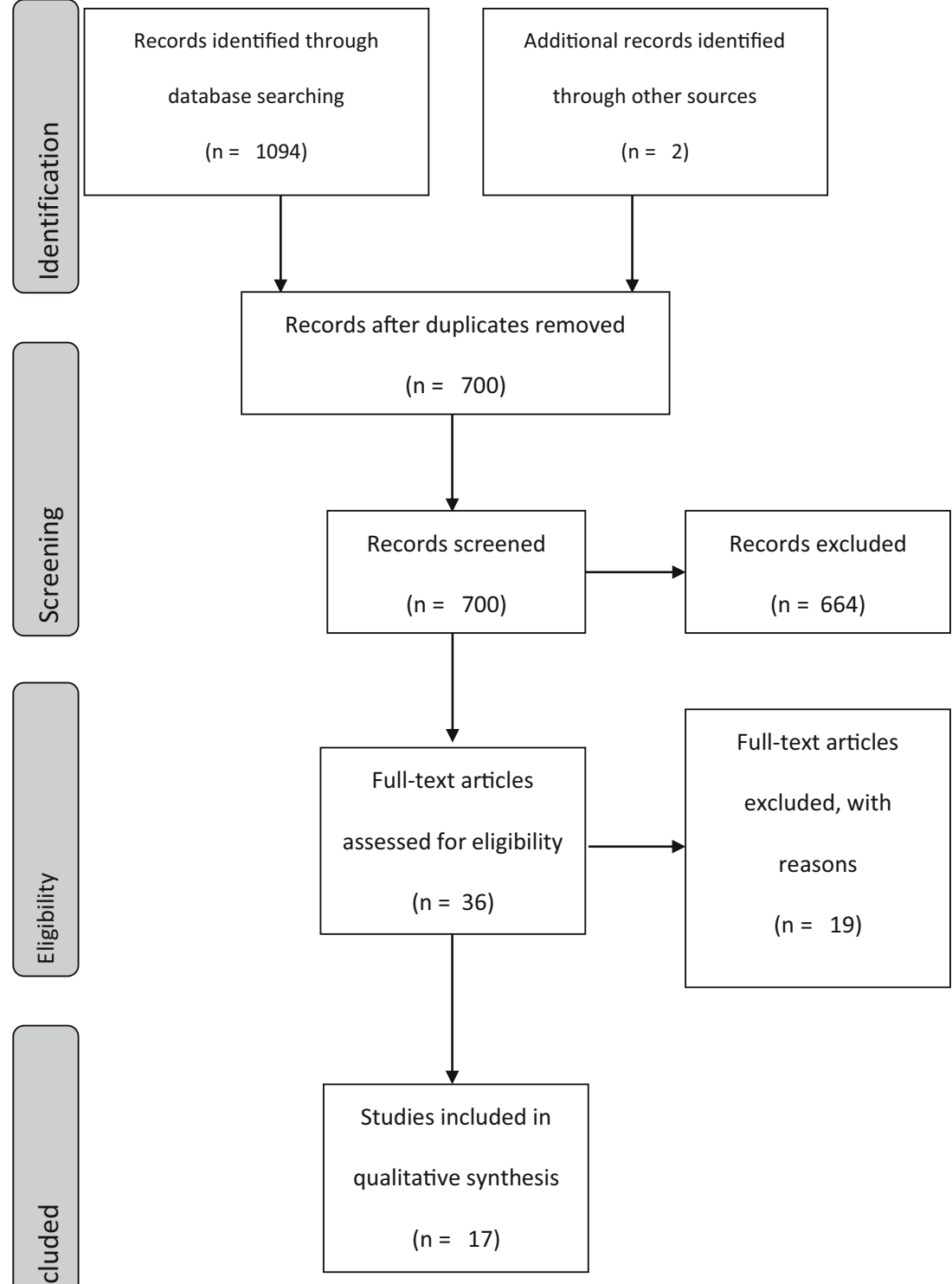

a normal height compared with the mean biparental predicted height at 14,15 , and 20 years $[36,38]$.

When compared with the WHO growth charts, ELBW infants seem to have a growth pattern almost similar to the 25 th percentile with some values around the 5 th percentile under the age of 1 . Only a few studies found a height that was comparable with the 50th percentile (Fig. 2).

\section{Weight}

Most studies agree that ELBW infants also remain lighter than NBW infants at each corrected age (CA) [17-21, 30, 37, 39-41]. However, two studies did not find a statistical significance between former ELBW and
NBW infants at the age of 14, 20 [36], and 34 [35]. One study described a lower weight in male patients only at the age of 11 [41] and one in female patients only at the age of 14.7 [20].

In their first 7 years of life, ELBW infants seem to have a weight similar to the 25 th percentile of the WHO growth charts, with a trend toward the 50th percentile afterwards (Fig. 3).

\section{Head circumference}

All studies investigating $\mathrm{HC}$ report that ELBW infants and adolescents have a smaller HC than NBW control peers in their childhood as well as when they reach adult age $[18,19$, 30, 37, 39-41]. 
Table 2 Overview of included studies

\begin{tabular}{|c|c|c|c|c|c|c|c|}
\hline $\begin{array}{l}\text { Authors and } \\
\text { reference }\end{array}$ & Study design & Group & $\begin{array}{l}\mathrm{N} \\
\text { (male/female) }\end{array}$ & Age $(\mathrm{N})$ at assessment & $\begin{array}{l}\text { Mean GA } \\
\text { (weeks) }\end{array}$ & Mean BW (grams) & $\begin{array}{l}\text { Measuring } \\
\text { methods }\end{array}$ \\
\hline $\begin{array}{l}\text { Atkinson SA et al. } \\
{[34]}\end{array}$ & $\begin{array}{l}\text { Longitudinal } \\
\text { cohort }\end{array}$ & $\begin{array}{l}\text { ELBW } \\
\text { NBW }\end{array}$ & $\begin{array}{l}125 \\
119\end{array}$ & $\begin{array}{l}23.1 \pm 1.4 \mathrm{y}(96) \\
23.5 \pm 1.2 \mathrm{y}(92)\end{array}$ & $\begin{array}{l}7.1 \pm 2.3 \\
\mathrm{NA}\end{array}$ & $\begin{array}{l}841 \pm 125 \\
\text { NA }\end{array}$ & DEXA \\
\hline Crane JD et al. [35] & $\begin{array}{l}\text { Cross-sectional } \\
\text { cohort }\end{array}$ & $\begin{array}{l}\text { ELBW } \\
\text { NBW }\end{array}$ & $\begin{array}{l}29(12 / 17) \\
16(7 / 9)\end{array}$ & $\begin{array}{l}34.3 \pm 0.33 \mathrm{y} \\
34.9 \pm 0.32 \mathrm{y}\end{array}$ & $\begin{array}{l}\text { NA } \\
\text { NA }\end{array}$ & $\begin{array}{l}830 \pm 20 \\
3330 \pm 100\end{array}$ & $\begin{array}{l}\text { Harpenden } \\
\quad \text { stadiometer } \\
\text { Electronic scale } \\
\text { MRI; DEXA }\end{array}$ \\
\hline $\begin{array}{l}\text { Doyle LW et al. } \\
\text { [36] }\end{array}$ & $\begin{array}{l}\text { Longitudinal } \\
\text { cohort }\end{array}$ & ELBW & $42(15 / 27)$ & $\begin{array}{l}\text { Birth to } 20.3 \pm 1.0 y \\
\text { at } 2 y, 5 y, 8 y, 14 y\end{array}$ & $27.4 \pm 2.0$ & $877 \pm 86$ & $\begin{array}{l}\text { Harpenden } \\
\quad \text { stadiometer } \\
\text { Digital scale }\end{array}$ \\
\hline Hack. et al. [20] & $\begin{array}{l}\text { Longitudinal } \\
\text { cohort }\end{array}$ & $\begin{array}{l}\text { ELBW } \\
\text { NBW }\end{array}$ & $\begin{array}{l}148(52 / 96) \\
176(65 / 111)\end{array}$ & $\begin{array}{l}8 \mathrm{y}(147) ; 14 \mathrm{y}(148) \\
8 \mathrm{y}(176) ; 14 \mathrm{y}(115)\end{array}$ & $\begin{array}{l}26.5 \pm 2 \\
\geq 37\end{array}$ & $\begin{array}{l}825 \pm 119 / 813 \pm 124 \\
3323 \pm 597 / 3238 \pm 411\end{array}$ & $\begin{array}{l}\text { Infantometer; } \\
\text { stadiometer }\end{array}$ \\
\hline Hill AS et al. [37] & $\begin{array}{l}\text { Retrospective } \\
\text { cohort }\end{array}$ & ELBW & 46 & $\begin{array}{l}\text { Birth; discharge; } 6 \mathrm{~m} \\
12 \mathrm{~m} ; 18 \mathrm{~m}\end{array}$ & $27.03 \pm 1.75$ & $796.22 \pm 140.18$ & NA \\
\hline Hirata T et al. [38] & $\begin{array}{l}\text { Longitudinal } \\
\text { cohort }\end{array}$ & ELBW & 103 & $\begin{array}{l}\text { Follow-up from birth to } \\
\qquad \begin{array}{l}14.3 \pm 1.8 \\
(8) \mathrm{y} / 15.6 \pm 1.5 \mathrm{y}(15)\end{array}\end{array}$ & $26.6 \pm 1.4$ & $818 \pm 110$ & $\begin{array}{l}\text { Standard balance } \\
\text { beam weight } \\
\text { scale with height } \\
\text { rod }\end{array}$ \\
\hline $\begin{array}{l}\text { Jordan IM et al. } \\
\text { [31] }\end{array}$ & $\begin{array}{l}\text { Retrospective } \\
\text { cohort }\end{array}$ & ELBW & $159(82 / 77)$ & $\begin{array}{l}\text { Birth, TCA, } 3 \mathrm{~m}, 9 \mathrm{~m}, 18 \mathrm{~m} \text {, } \\
\quad 36 \mathrm{~m}\end{array}$ & $28 \pm 2$ & $851.2 \pm 116.5$ & NA \\
\hline Kwinta $P$ et al. [39] & $\begin{array}{l}\text { Cross-sectional } \\
\text { cohort }\end{array}$ & $\begin{array}{l}\text { ELBW } \\
\text { NBW }\end{array}$ & $\begin{array}{l}81(29 / 52) \\
39(19 / 20)\end{array}$ & $\begin{array}{l}6.7 \pm 0.4 y \\
6.9 \pm 0.8 y\end{array}$ & $\begin{array}{l}27.2 \pm 2.1 \\
39.9 \pm 1.4\end{array}$ & $\begin{array}{l}845 \pm 130 \\
3554 \pm 512\end{array}$ & $\begin{array}{l}\text { Multifrequency } \\
\text { bioimpedance }\end{array}$ \\
\hline Lin YC et al. [40] & $\begin{array}{l}\text { Retrospective } \\
\text { cohort }\end{array}$ & ELBW & $100(41 / 59)$ & $6 \mathrm{~m} \mathrm{(68),} 12 \mathrm{~m}(67), 24 \mathrm{~m}(62)$ & $26 *$ & $772 *$ & Standard procedure \\
\hline Mól N et al. [41] & $\begin{array}{l}\text { Prospective } \\
\text { cohort }\end{array}$ & $\begin{array}{l}\text { ELBW } \\
\text { NBW }\end{array}$ & $\begin{array}{l}81(29 / 52) \\
36(19 / 17)\end{array}$ & $\begin{array}{l}6.61 \pm 0.36 \mathrm{y}(81) ; 11.06 \pm 0.38 \mathrm{y} \\
\quad(62) \\
6.98 \pm 0.83 \mathrm{y} ; 10.62 \pm 0.82 \mathrm{y}\end{array}$ & $\begin{array}{l}27.3 \pm 2.3 \\
39.8 \pm 1.4\end{array}$ & $\begin{array}{l}843.3 \pm 132.4 \\
3589.4 \pm 538.8\end{array}$ & $\begin{array}{l}\text { Standard medical } \\
\text { tape; fixed } \\
\text { stadiometer } \\
\text { Medical scale } \\
\quad \text { (nearest } 100 \mathrm{~g} \text { ) }\end{array}$ \\
\hline $\begin{array}{l}\text { Monset-Couchard } \\
\text { M et al. [22] }\end{array}$ & $\begin{array}{l}\text { Longitudinal } \\
\text { cohort }\end{array}$ & ELBW & $166(64 / 102)$ & $\begin{array}{l}\text { Consistent follow-up from birth } \\
\text { to } 18 \text { years of age }\end{array}$ & NA & NA & NA \\
\hline $\begin{array}{l}\text { Morrison } \mathrm{M} \text { et al. } \\
\text { [17] }\end{array}$ & $\begin{array}{l}\text { Prospective } \\
\text { cohort }\end{array}$ & $\begin{array}{l}\text { ELBW } \\
\text { NBW }\end{array}$ & $\begin{array}{l}100(40 / 60) \\
89(37 / 52)\end{array}$ & $\begin{array}{l}31.63 \pm 1.66 \mathrm{y} \\
31.96 \pm 1.42 \mathrm{y}\end{array}$ & $\begin{array}{l}27.10 \pm 2.45 \\
\text { NA }\end{array}$ & $\begin{array}{l}829.00 \pm 130.38 \\
3391.30 \pm 442.28\end{array}$ & $\begin{array}{l}\text { Harpenden } \\
\quad \text { stadiometer } \\
\text { Electronic scale } \\
\text { DEXA }\end{array}$ \\
\hline $\begin{array}{l}\text { Peralta-Carcelen M } \\
\text { et al. [18] }\end{array}$ & $\begin{array}{l}\text { Cross-sectional } \\
\text { cohort }\end{array}$ & $\begin{array}{l}\text { ELBW } \\
\text { NBW }\end{array}$ & $\begin{array}{l}53(22 / 31) \\
53\end{array}$ & $\begin{array}{l}14.8 \pm 1.8 \mathrm{y} \\
14.9 \pm 1.7 \mathrm{y}\end{array}$ & $\begin{array}{l}28.2 \pm 2.3 \\
>37\end{array}$ & $\begin{array}{l}849 \pm 109 \\
3355 \pm 526\end{array}$ & DEXA \\
\hline $\begin{array}{l}\text { Raaijmakers A } \\
\text { et al. [30] }\end{array}$ & $\begin{array}{l}\text { Longitudinal } \\
\text { case-control }\end{array}$ & $\begin{array}{l}\text { ELBW } \\
\text { NBW }\end{array}$ & $\begin{array}{l}93(49 / 44) \\
87(43 / 44)\end{array}$ & $\begin{array}{l}\text { Birth (140); } 9 \mathrm{~m} \mathrm{(118);24} \mathrm{m} \\
\quad(96) ; 11.3 \pm 1.4 \mathrm{y}(93) \\
10.9 \pm 1.3 \mathrm{y}\end{array}$ & NA & NA & $\begin{array}{l}\text { Bioelectrical } \\
\text { impedance }\end{array}$ \\
\hline $\begin{array}{l}\text { Rodríguez-Soriano } \\
\text { J et al. [21] }\end{array}$ & $\begin{array}{l}\text { Longitudinal } \\
\text { cohort }\end{array}$ & $\begin{array}{l}\text { ELBW } \\
\text { NBW }\end{array}$ & $\begin{array}{l}40(23 / 17) \\
43(25 / 18)\end{array}$ & $\begin{array}{l}8.6 \pm 1.8 \mathrm{y} \\
8.5 \pm 1.8 \mathrm{y}\end{array}$ & $\begin{array}{l}27.6^{*} \\
\text { NA }\end{array}$ & $\begin{array}{l}845^{*} \\
\text { NA }\end{array}$ & $\begin{array}{l}\text { Harpenden } \\
\text { stadiometer }\end{array}$ \\
\hline Saigal $S$ et al. [19] & $\begin{array}{l}\text { Prospective } \\
\text { cohort }\end{array}$ & ELBW & $147(65 / 82)$ & $\begin{array}{l}\text { Birth (179); 1y (157); 2y (137); } \\
\quad 3 \mathrm{y}(123) ; 8 \mathrm{y}(147) ; 14.1 \pm 1.6 \mathrm{y} \\
\quad(144) ; 23.3 \pm 1.2 \mathrm{y}(147) \\
8 \mathrm{y}(145) ; 141.4 \pm 1.3(121) ; \\
\quad 23.6 \pm 1.1 \mathrm{y}(131)\end{array}$ & $27.1 \pm 2.3$ & $3380 \pm 475$ & $\begin{array}{l}\text { Calibrated scale } \\
\text { Balance beam scale }\end{array}$ \\
\hline Sices L et al. [42] & $\begin{array}{l}\text { Retrospective } \\
\text { cohort }\end{array}$ & ELBW & $154(71 / 83)$ & $\begin{array}{l}\text { Birth (154); } 39.2 \pm 3.0 \mathrm{w}(154) \\
\quad 4.4 \pm 0.7 \mathrm{~m}(143) ; 8.6 \pm 1.0 \mathrm{~m} \\
\quad(138) ; 19.0 \pm 1.2 \mathrm{~m} \mathrm{(151)}\end{array}$ & $25.9 \pm 1.8$ & $768 \pm 140$ & $\begin{array}{l}\text { Harpenden } \\
\text { infantemeter } \\
\text { Health-o-Meter } \\
\text { Scale }\end{array}$ \\
\hline
\end{tabular}

$\mathrm{N}$ is the number of infants in the selected cohort used to determine the mean GA and BW

$\mathrm{N}$ at age of assessment was added when different from the initial cohort

$E L B W$ extremely low birth weight, $N B W$ normal birth weight, $G A$ gestational age, $w$ weeks, $m$ months, $y$ years $B W$ birth weight, $N A$ not available; information could not be extracted from the study, $D E X A$ dual-energy x-ray absorptiometry

* Standard deviation scores are missing in the study

The WHO growth charts only describe $\mathrm{HC}$ until the age of 5. During this period, the HC of ELBW infants seems to fluctuate between the 25th and 50th percentile with a trend toward the 25 th percentile (Fig. 4).

\section{Body mass index}

Since both their height and weight are lower than that of NBW children, it is still unclear whether former ELBW 


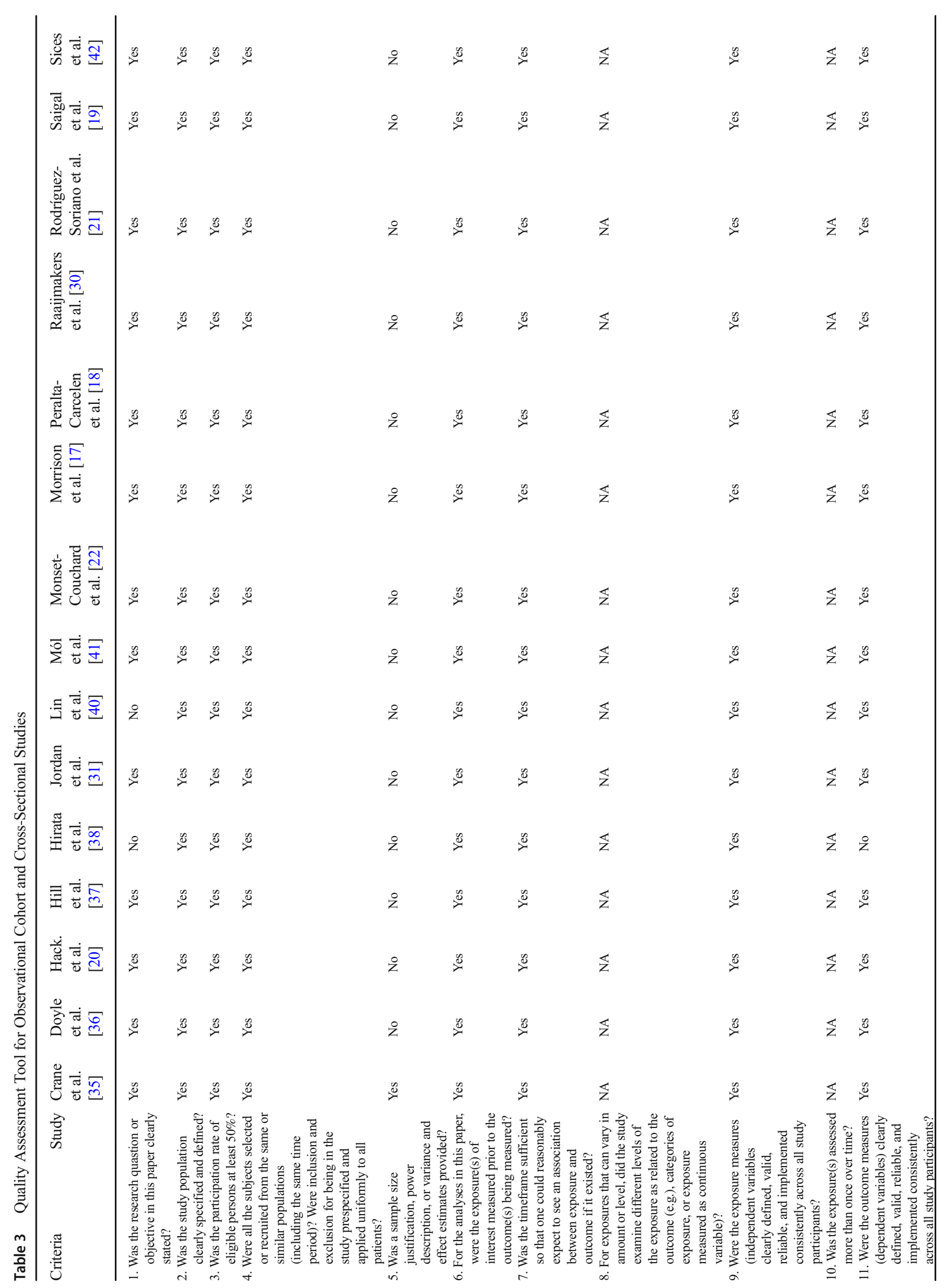




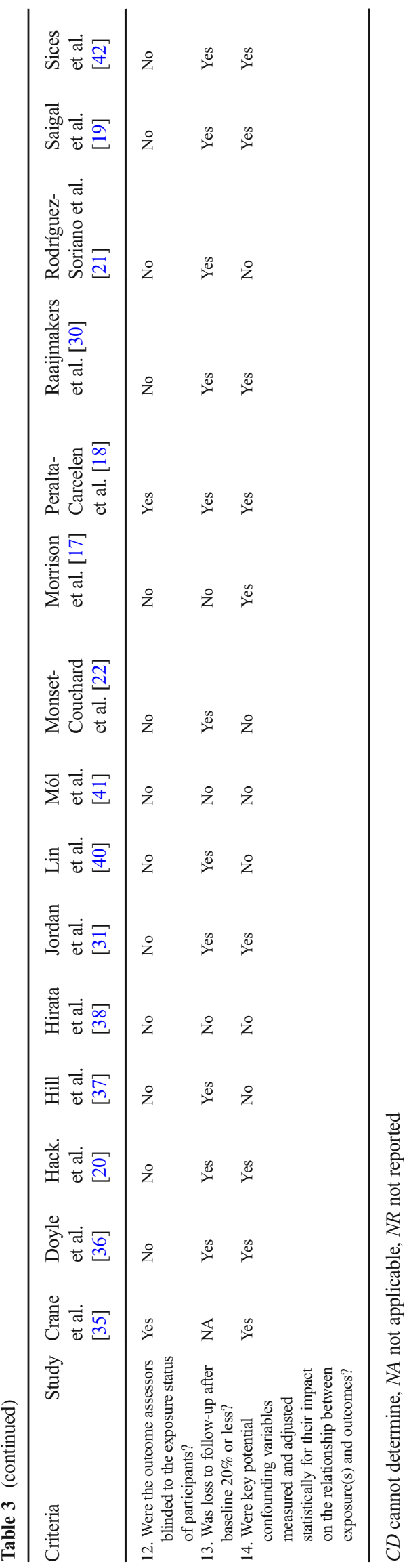

children achieve a BMI score that is equal to that of NBW children and adolescents [17, 19, 20, 36], or if their BMI will be lower $[21,30,32,39,41]$. One study found a higher BMI score in ELBW infants, but this result did not reach statistical significance when compared to NBW control subjects [35].

Studies do not contain BMI data under the age of 6.7. By age 8 years and afterwards, BMI scores of former ELBW infants seem to reach values between the 50th and the 75 th percentile of the WHO growth charts (Fig. 5).

\section{Catch-up growth patterns}

Studies investigating catch-up growth commonly report that ELBW infants experience a period of neonatal growth failure from birth to TCA, which sometimes continues until 8 months or even after 1 year of age. This growth failure is followed by a period of catch-up growth, especially for weight and height, which continues until adulthood [19, 20, 22, 35-38, 41, 42].

Studies do not uniformly agree on the time of onset of catch-up growth for different growth parameters and the use of different definitions makes it hard to compare the results. Some found catch-up growth starting from the age of 2 years [19], while others report catch-up growth in most subjects before the age of 3 years [22, 31, 42].

Former ELBW infants seem to have a marked catch-up growth in weight and height between the age of 8 and 14 years, with a more pronounced increment in weight than in height. During this period, there was also a marked catch-up growth for BMI [19, 20, 36].

It is suggested that catch-up growth for weight and height in the first 2 years of life or $\mathrm{HC}$ in the first 9 months is associated with a lower \% TBF at adolescence. Catch-up growth for weight during childhood (2-11 years) was also associated with a lower \% TBF at young adolescence [30]. Hack et al. however discuss that catch-up growth throughout infancy is associated with measures of obesity at the age of 14 and is therefore not beneficial [20].

\section{SGA versus $A G A$}

As the definition is based on a weight, a relevant portion of ELBW infants are SGA, higher than infants born with a NBW [18, 19]. After the age of 8.6 and afterwards, no significant difference in height and weight remained between SGA and AGA infants, but SGA infants reached a height significantly below target height at 11 years [21]. Only one study found a significantly smaller HC in SGA ELBW infants compared to AGA ELBW infants at the age of 14 [18]. 


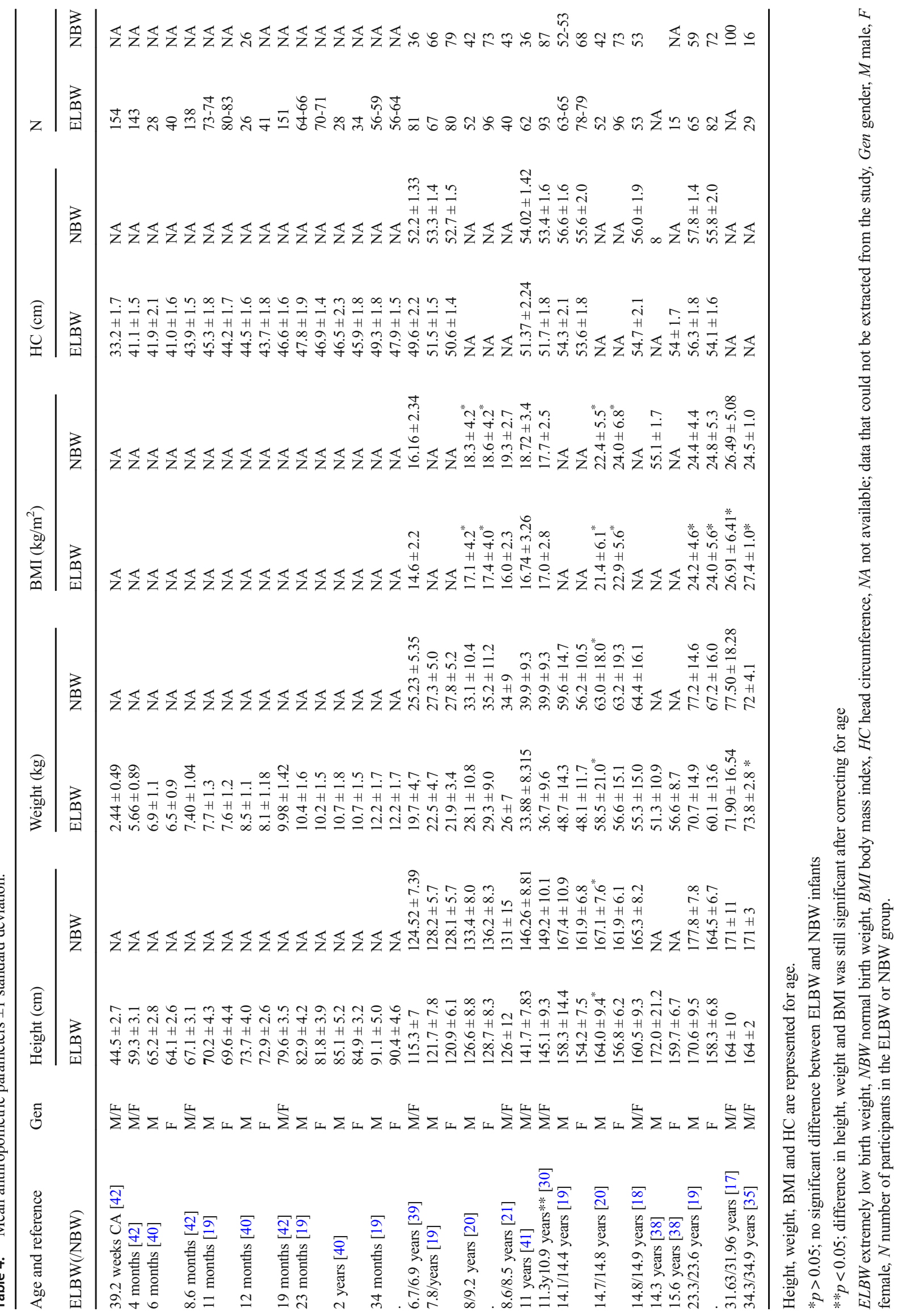




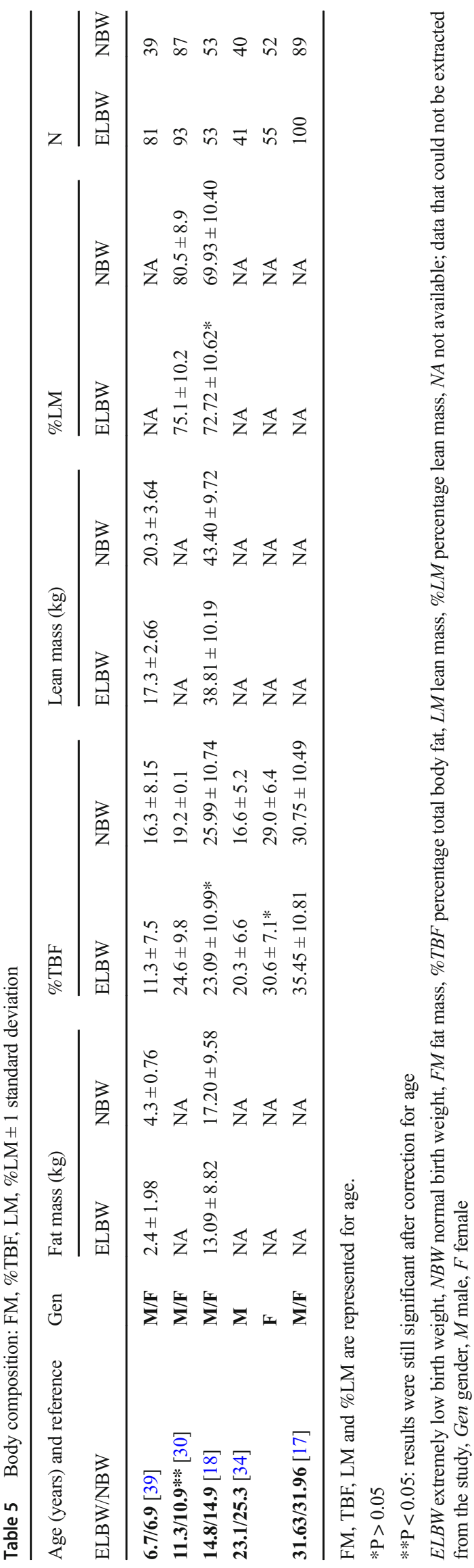

\section{Body composition}

\section{FM, LM, and relative body composition}

Two studies report a lower \%LM or lean mass index (LMI, i.e., total lean mass $/$ height $^{2}$ ), with a higher $\% \mathrm{TBF}$ and fat mass index (FMI, i.e., total fat mass/height ${ }^{2}$ ) in ELBW infants [17, 30]. The third study found a higher $\% \mathrm{TBF}$ in male infants only [34]. Otherwise, a fourth study found a significantly lower absolute LM and FM, but also a lower \% TBF in children born with an ELBW at the age of 7 [39].

The fifth study found no significant difference in \% TBF and \%LM between ELBW and NBW infants reaching the age of 14. They conclude that ELBW infants have a lower FM and $\mathrm{LM}$, but that the relative body composition is similar to those of NBW children [18] (Table 5).

There was no difference in body composition between SGA and AGA ELBW infants [18, 30].

\section{Fat distribution}

Only one study and one abstract reported on fat distribution in ELBW infants with different results since ELBW infants had a higher portion of visceral adipose tissue deposition in one study [34], and this higher portion was limited to the subcutaneous fat areas only in the other study [35]. The last report also found higher proportions of pancreatic and liver fat in ELBW infants reaching young adulthood, but an ELBW was not an independent influencer of these fat fractions [35].

\section{Body water}

Two studies investigated body water in ELBW infants, with contradictory results. One study found lower absolute TBW but a higher \% TBW in former ELBW infants [39], while the other found no significant difference in \% TBW between ELBW and NBW infants [30].

\section{Discussion}

Children born as ELBW infants have a specific growth pattern until birth and exhibit a different body composition at birth and TCA than their NBW counterparts. Their compromised growth may result in a body composition later in life that differs from NBW children. Literature describing growth and evolution of body composition after hospital discharge and in later life, however, is scarce. Therefore, we performed a systematic literature search regarding growth parameters and body composition in former ELBW infants throughout childhood and adolescence.

Most studies state that ELBW infants remain smaller and lighter than NBW infants at every stage of their 


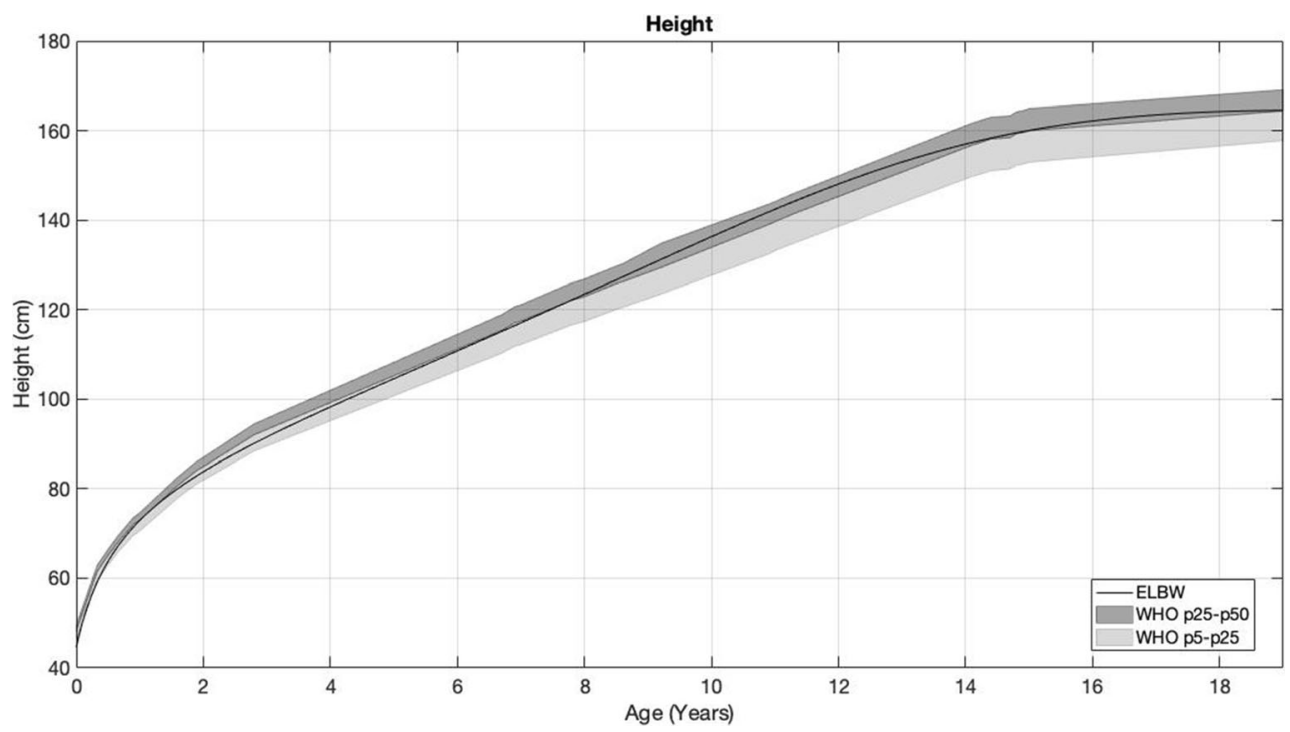

Fig. 2 Height for age. Height for age is plotted for ELBW infants. The shaded areas represent the 5th-25th and 25-50th percentiles of the WHO growth charts;ELBW function: General model Rat44: $\mathrm{f}(\mathrm{x})=\left(\mathrm{p} 1 * \mathrm{x}^{\wedge} 4+\right.$ $\left.\mathrm{p} 2 * \mathrm{x}^{\wedge} 3+\mathrm{p} 3 * \mathrm{x}^{\wedge} 2+\mathrm{p} 4 * \mathrm{x}+\mathrm{p} 5\right) /,\left(\mathrm{x}^{\wedge} 4+\mathrm{q} 1 * \mathrm{x}^{\wedge} 3+\mathrm{q} 2 \mathrm{x}^{\wedge} 2+\mathrm{q} 3 * \mathrm{x}+\mathrm{q} 4\right)$ Coefficients (with $95 \%$ confidence bounds): $1=204.5(-159.7$, $568.8), \mathrm{p} 2=-2524(-1.05 \mathrm{e}+04,5452), \mathrm{p} 3=-2966(-2.332 \mathrm{e}+05$,

development. In two studies, ELBW infants attained their predicted biparental target height [36, 38]. Although catch-up growth is often described, ELBW infants still attain lower growth parameters by the time they reach adulthood. Since their growth restriction is expressed both in weight and height, it is not clear whether their BMI at
$2.272 \mathrm{e}+05), \mathrm{p} 4=5.533 \mathrm{e}+05(-4.751 \mathrm{e}+06,5.858 \mathrm{e}+06), \mathrm{p} 5=1.924 \mathrm{e}+05$ $(-1.825 \mathrm{e}+06,2.21 \mathrm{e}+06), \mathrm{q} 1=2.602(-197.4,202.6), \mathrm{q} 2=-432.9$ $(-5454,4588), \mathrm{q} 3=6270(-5.07 \mathrm{e}+04,6.324 \mathrm{e}+04), \mathrm{q} 4=4312$ $(-4.087 \mathrm{e}+04,4.95 \mathrm{e}+04)$, Goodness of fit: SSE: $36.72 ; R^{2}: 0.999$; Adjusted $R^{2}$ : 0.9984; RMSE: 1.62 ; SSE sum of squared estimate of errors, $R M S E$ root mean square error

teen and adult age significantly differs from that of NBW infants or not. It is suggested that catch-up growth is more pronounced for weight than for height ("stunting"), resulting in infants being proportionally heavier for their height, although former ELBW infants are never assumed to have a significantly higher BMI score $[20,32,36]$.

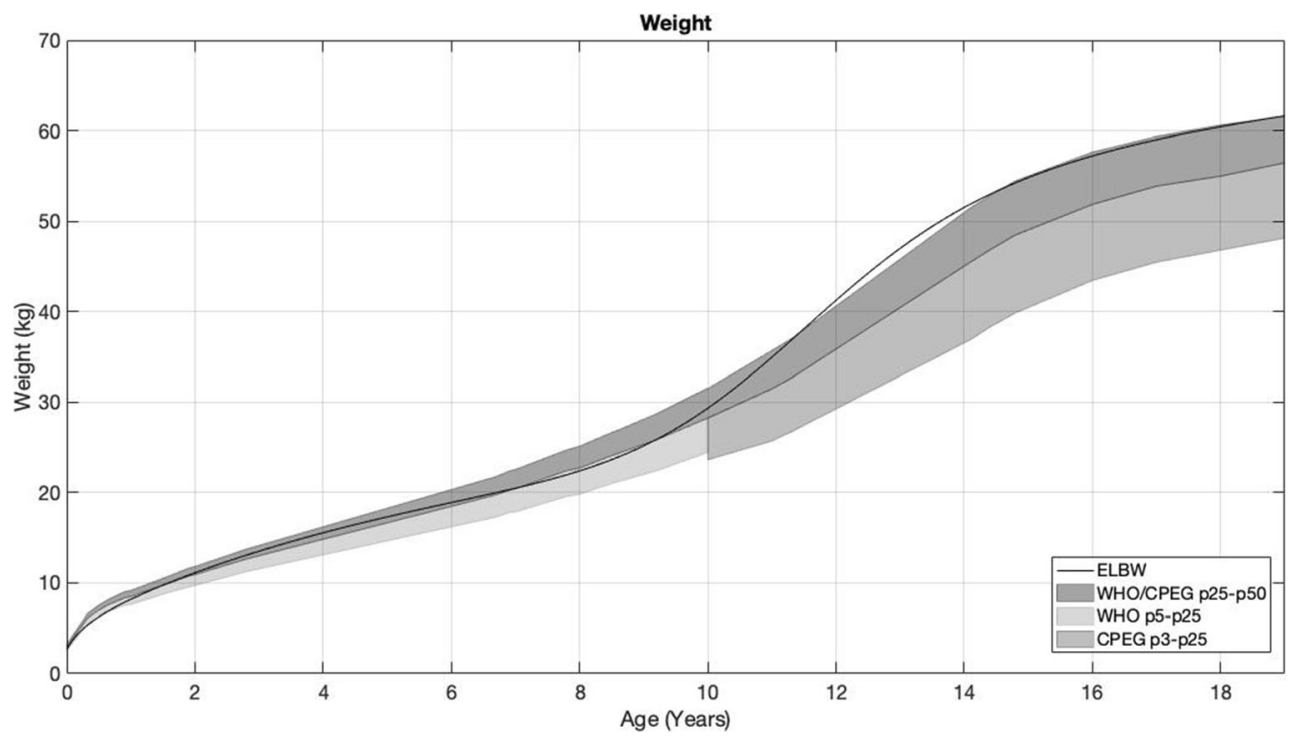

Fig. 3 Weight for age. Weight for age is plotted for ELBW infants. The shaded areas represent the 5 th -25 th and 25 th- 50 th percentiles of the WHO/CPEG growth charts and the 3th-25th percentiles of the extended charts provided by the CPEG; ELBW function: General model Rat45: $\mathrm{f}(\mathrm{x})=\left(\mathrm{p} 1 * \mathrm{x}^{\wedge} 4+\mathrm{p} 2 * \mathrm{x}^{\wedge} 3+\mathrm{p} 3 * \mathrm{x}^{\wedge} 2+\mathrm{p} 4 * \mathrm{x}+\mathrm{p} 5\right) / ;\left(\mathrm{x}^{\wedge} 5+\mathrm{q} 1{ }^{*} \mathrm{x}^{\wedge} 4+\right.$ $\left.\mathrm{q} 2 * \mathrm{x}^{\wedge} 3+\mathrm{q} 3 * \mathrm{x}^{\wedge} 2+\mathrm{q} 4 * \mathrm{x}+\mathrm{q} 5\right)$; where $\mathrm{x}$ is normalized by mean 9.043 and std. 10.21; Coefficients (with $95 \%$ confidence bounds): $\mathrm{p} 1=$ $5.146 \mathrm{e}+06(-3.084 \mathrm{e}+11,3.084 \mathrm{e}+11) ; \mathrm{p} 2=9.356 \mathrm{e}+06(-5.607 \mathrm{e}+11$,
$5.607 \mathrm{e}+11) ; \mathrm{p} 3=4.198 \mathrm{e}+06(-2.516 \mathrm{e}+11,2.516 \mathrm{e}+11) ; \mathrm{p} 4=9.184 \mathrm{e}+05$ $(-5.504 \mathrm{e}+10,5.505 \mathrm{e}+10) ; \mathrm{p} 5=8.684 \mathrm{e}+05(-5.204 \mathrm{e}+10,5.204 \mathrm{e}+10) ;$ $\mathrm{q} 1=4.386 \mathrm{e}+04(-2.628 \mathrm{e}+09,2.628 \mathrm{e}+09) ; \mathrm{q} 2=1.847 \mathrm{e}+05(-1.107 \mathrm{e}+$ $10,1.107 \mathrm{e}+10) ; \mathrm{q} 3=7.957 \mathrm{e}+04(-4.768 \mathrm{e}+09,4.768 \mathrm{e}+09) ; \mathrm{q} 4=$ $-1.152 \mathrm{e}+04(-6.898 \mathrm{e}+08,6.898 \mathrm{e}+08) ; \mathrm{q} 5=3.429 \mathrm{e}+04(-2.055 \mathrm{e}+09$, $2.055 \mathrm{e}+09)$; Goodness of fit: SSE: $32.65 ; R^{2}$ : 0.9972; Adjusted $R^{2}$ : 0.9949; RMSE: 1.723; SSE sum of squared estimate of errors, RMSE root mean square error 


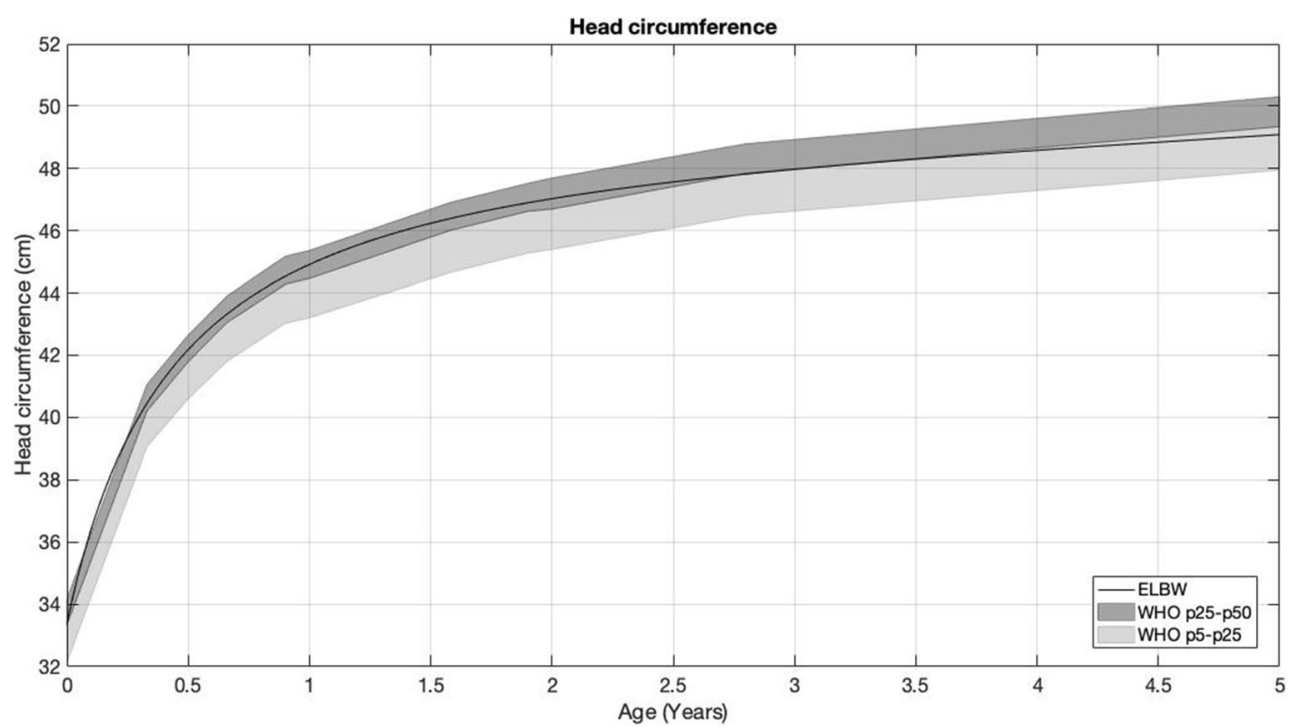

Fig. 4 Head circumference for age. Head circumference for age is plotted for ELBW infants. The shaded areas represent the 5th-25th and 25-50th percentiles of the WHO growth charts; ELBW function: General model Rat24: $f(x)=\left(p 1^{*} x^{\wedge} 2+p 2 * x+p 3\right) / ;\left(x^{\wedge} 4+q 1^{*} x^{\wedge} 3+q 2 * x^{\wedge} 2+q 3^{*} x+\right.$ q4); Coefficients (with 95\% confidence bounds): $1=-4439(-3.263 \mathrm{e}+$ $05,3.174 \mathrm{e}+05) ; \mathrm{p} 2=1.478 \mathrm{e}+06(-4.638 \mathrm{e}+06,7.593 \mathrm{e}+06) ; \mathrm{p} 3=4.502 \mathrm{e}+$

Former ELBW infants do not only reach a lower target height and weight at every CA but they also have a smaller $\mathrm{HC}$. It has been suggested that a smaller $\mathrm{HC}$ is related to poorer cognitive outcomes [43-45], but one study investigating neurodevelopment did not find an association between catch-up growth for $\mathrm{HC}$ and neurocognitive outcome [30].

When comparing with the WHO growth charts, we came to similar results, especially for height and HC. ELBW infants
$05(-1.222 \mathrm{e}+06,2.123 \mathrm{e}+06) ; \mathrm{q} 1=32.91(-70.88,5.063) ; \mathrm{q} 2=27.98$ $(-7218,7274) ; \mathrm{q} 3=2.934 \mathrm{e}+04(-9.444 \mathrm{e}+04,1.531 \mathrm{e}+05) ; \mathrm{q} 4=1.349 \mathrm{e}+$ $04(-3.662 \mathrm{e}+04,6.359 \mathrm{e}+04)$; Goodness of fit: SSE: $5.773 ; R^{2}: 0.99$; Adjusted $R^{2}$ : 0.9845; RMSE: 0.7244; SSE sum of squared estimate of errors, $R M S E$ root mean square error

seem to attain a height around the 25 th percentile at young adult age. Their HC also seems to be around the 25 th percentile. In the first 7 years of life, their weight and BMI remain around the 25 th percentile with afterwards a trend toward the 50 th percentile. Their BMI seems to reach values between the 50th and the 75th percentile by young adulthood.

In the studies that linked catch-up growth to adverse health outcomes, there is no consensus whether early catch-up growth is

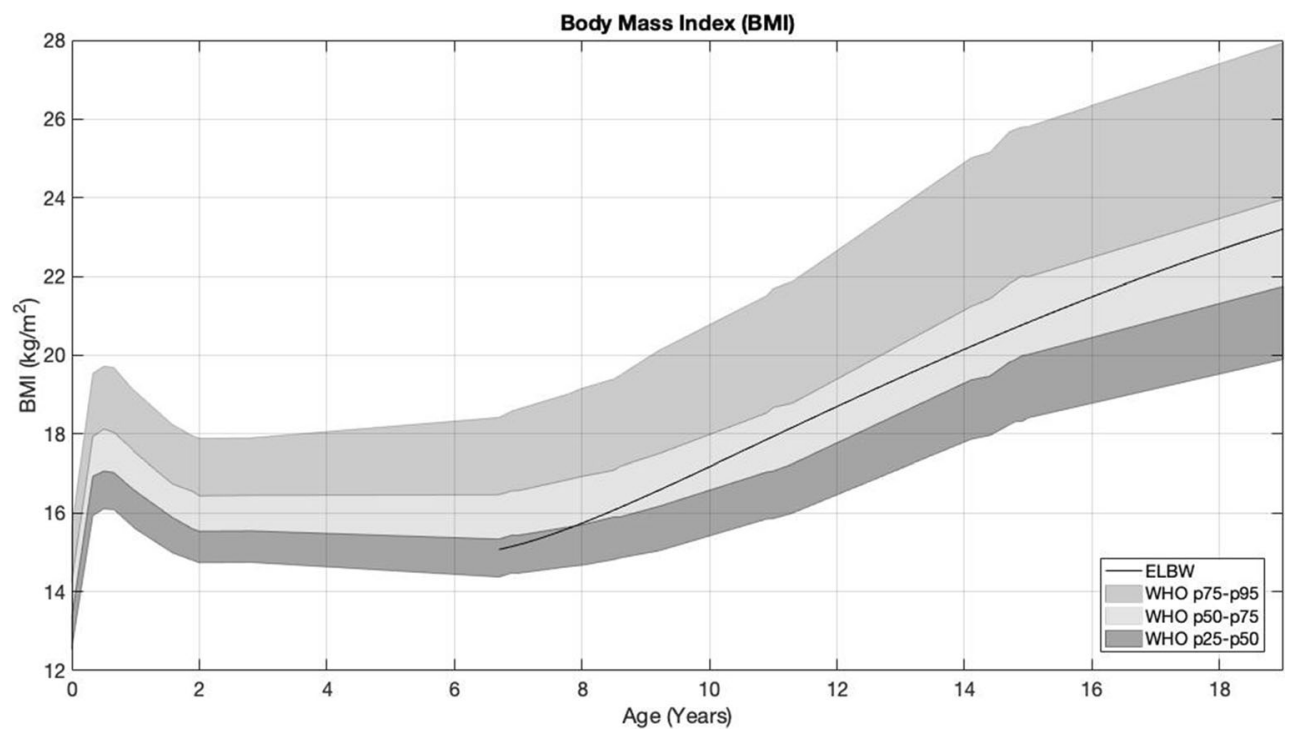

Fig 5 BMI for age. BMI for age is plotted for ELBW infants. The shaded areas represent the 25th-50th, 50-75th, and 75th-95th percentiles of the WHO growth charts; ELBW function: General model Rat34: $\mathrm{f}(\mathrm{x})=($ $\left.\mathrm{p} 1 * \mathrm{x}^{\wedge} 3+\mathrm{p} 2 * \mathrm{x}^{\wedge} 2+\mathrm{p} 3 * \mathrm{x}+\mathrm{p} 4\right) / ;\left(\mathrm{x}^{\wedge} 4+\mathrm{q} 1{ }^{*} \mathrm{x}^{\wedge} 3+\mathrm{q} 2 * \mathrm{x}^{\wedge} 2+\mathrm{q} 3 * \mathrm{x}+\mathrm{q} 4\right) ;$ Coefficients (with $95 \%$ confidence bounds): $\mathrm{p} 1=2361(-1.199 \mathrm{e}+06$, $1.203 \mathrm{e}+06) ; \mathrm{p} 2=87.87(-4.044 \mathrm{e}+11,4.044 \mathrm{e}+11) ; \mathrm{p} 3=857.6$
$(-1.361 \mathrm{e}+11,1.361 \mathrm{e}+11) ; \mathrm{p} 4=225.7(-1.909 \mathrm{e}+11,1.909 \mathrm{e}+11) ; \mathrm{q} 1=$ $7.852(-1.713 \mathrm{e}+08,1.713 \mathrm{e}+08) ; \mathrm{q} 2=1665(-1.279 \mathrm{e}+09,1.279 \mathrm{e}+09)$; $\mathrm{q} 3=-4398(-2.848 \mathrm{e}+11,2.848 \mathrm{e}+11) ; \mathrm{q} 4=-1901(-8.607 \mathrm{e}+11$, 8.607e+11); Goodness of fit: SSE: $9.316 ; R^{2}$ : 0.9557; Adjusted $R^{2}$ : 0.8008; RMSE: 2.158; SSE sum of squared estimate of errors, RMSE root mean square error 
beneficial for metabolic and cardiovascular diseases, hypertension, and renal function later in life or not [12, 19, 20, 30].

With regard to body composition and fat distribution in ELBW infants, there remains some controversy. Studies do not agree on differences in \%LM, FM, \% TBF and fat distribution $[17,18,30,34,35,39]$.

It is suggested that an aberrant body composition with a higher \% TBF could indeed make ELBW infants and adolescents more susceptible to cardiovascular diseases and insulin resistance [17, 30, 34].

Nevertheless, there is a lack in information about the body composition in former ELBW infants and it is possible that differences in body composition and fat distribution only appear with advancing age, thus there is a need for longitudinal studies [17]. There is also no agreement on the ideal measure method to investigate body composition.

There are some limitations to this review as a result of the inclusion of long-term follow-up studies and the comparison of cases at different points of time.

Some of the study patients were born in the 1990s or earlier. Perinatal care and nutritional practices have since evolved, so one should be careful extrapolating these results to the current population of ELBW neonates [17, 18].

Another consequence of including longitudinal follow-up studies is a certain degree of loss of follow-up for part of the subjects, which could cause bias in the remaining study population.

Furthermore, because we did not have access to individual growth data in the different studies, we focused on mean data. Studies also use different definitions of SGA and often do not compare the GA of SGA and AGA infants. This is an import limitation, as SGA infants will have a higher GA per definition and might therefore exhibit a different growth pattern. Nevertheless, the results in most of the studies so far do not show a significant difference in growth parameters between SGA and AGA infants after the age of 8.6, but former SGA infants often represent the smallest spectrum of ELBW infants later in life. One more main limitation is the assessment at many different time points and the heterogeneity of the study populations.

Future studies should investigate the correlation between growth, weight gain, and adipose tissue development since not only ELBW but also BMI is suggested to be a possible influencer of body composition and related adverse health outcomes [17].

Intervention studies should focus on proportional growth and weight gain, especially from the age of 8 until the age of 14. However, increase in height and weight during this period might be influenced by inadequate growth in the first years of life. Also, catch-up growth for height and weight in the first 2 years of life might be beneficial for body composition later in life $[19,20,30,36,41]$. Careful follow-up throughout childhood is therefore needed to optimize the growth patterns of ELBW infants.

\section{Conclusion}

Studies consistently report that ELBW infants attain lower growth parameters than NBW control patients after hospital discharge throughout childhood and adolescence. Although there seems to be a certain degree of catch-up growth for all growth parameters, differences in anthropometric parameters continue to exist with advancing age. Since catch-up growth is often more pronounced for weight than for height, it is important to strive for proportional growth in intervention studies.

There is a lack of studies investigating body composition in ELBW infants. There is some evidence that they might have an aberrant body composition, but studies do not draw uniform conclusions. Associations between body composition and cardiovascular diseases are suggested. Further research and longitudinal studies are needed to ascertain whether the higher prevalence of metabolic and cardiovascular diseases could indeed be a consequence of an aberrant growth pattern, different body composition, or inadequate fat tissue development.

Authors' Contributions Both authors drafted the study and were involved in the study design. C.V.d.P. performed the systematic review. In case of uncertainties, the second author was consulted. Both authors wrote the final manuscript and approved the manuscript.

Funding There is no funding source.

\section{Compliance with ethical statements}

Ethical approval This article does not contain any studies with human participants or animals performed by any of the authors.

Conflict of interest The authors declare that they have no conflict of interest, and there was no specific funding for this project.

\section{References}

1. Tremblay G, Boudreau C, Bélanger S, St-Onge O, Pronovost E, Simonyan D, Marc I (2017) Body composition in very preterm infants: role of neonatal characteristics and nutrition in achieving growth similar to term infants. Neonatology 111:214-221. https:// doi.org/10.1159/000450882

2. Johnson MJ, Wootton SA, Leaf AA, Jackson AA (2012) Preterm birth and body composition at term equivalent age: a systematic review and meta-analysis. Pediatrics 130:e640-e649. https://doi. org/10.1542/peds.2011-3379

3. Cole TJ, Statnikov Y, Santhakumaran S, Pan H, Modi N (2014) Birth weight and longitudinal growth in infants born below 32 weeks' gestation: a UK population study. Arch Dis Child Fetal Neonatal Ed 99:F34-F40. https://doi.org/10.1136/archdischild2012-303536

4. Osmond C, Barker DJ (2000) Fetal, infant, and childhood growth are predictors of coronary heart disease, diabetes, and hypertension in adult men and women. Environ Health Perspect 108:545-553. https://doi.org/10.1289/ehp.00108s3545 
5. Euser AM, de Wit CC, Finken MJJ, Rijken M, Wit JM (2008) Growth of preterm born children. Horm Res Paediatr 70:319-328. https://doi.org/10.1159/000161862

6. Barker DJP (2006) Adult consequences of fetal growth restriction. Clin Obstet Gynecol 49:270-283. https://doi.org/10.1097/ 00003081-200606000-00009

7. Euser AM (2009) Long-term consequences of differences in early growth: epidemiological aspects. Retrieved from https:// openaccess.leidenuniv.nl/handle/1887/14485. Accessed 10 Dec 2019

8. Luyckx VA, Perico N, Somaschini M, Manfellotto D, Velensise H, Cetin I, Simeoni U, Allegaert K, Vikse BE, Steegers EA, Adu D, Montini G, Remuzzi G, Brenner BM, writing group of the Low Birth Weight and Nephron Number Working Group (2017) A developmental approach to the prevention of hypertension and kidney disease: a report from the low birth weight and nephron number working group. Lancet 390:424-428. https://doi.org/10.1016/ S0140-6736(17)30576-7

9. Ong KK, Kennedy K, Castañeda-Gutiérrez E, Forsyth S, Godfrey KM, Koletzko B, Latulippe ME, Ozanne SE, Rueda R, Schoemaker MH, van der Beek EM, van Buuren S, Fewtrell M (2015) Postnatal growth in preterm infants and later health outcomes: a systematic review. Acta Paediatr 104:974-986. https:// doi.org/10.1111/apa.13128

10. Barker DJ, Osmond C, Winter PD, Margetts B, Simmonds SJ (1989) Weight in infancy and death from ischaemic heart disease. Lancet 334:577-580. https://doi.org/10.1016/S0140-6736(89) 90710-1

11. Ong KK, Ahmed ML, Emmett PM, Preece MA, Dunger DB (2000) Association between postnatal catch-up growth and obesity in childhood: prospective cohort study [corrected] [published erratum appears in BR MED J 2000; 320(7244): 1244]. BMJ Br Med J (International Ed) 320:967-971. https://doi.org/10.1136/bmj.320. 7240.967

12. Fewtrell MS, Doherty C, Cole TJ, Stafford M, Hales CN, Lucas A (2000) Effects of size at birth, gestational age and early growth in preterm infants on glucose and insulin concentrations at 9-12 years. Diabetologia 43:714-717. https://doi.org/10.1007/s001250051368

13. Parkinson JRC, Hyde MJ, Gale C, Santhakumaran S, Modi N (2013) Preterm birth and the metabolic syndrome in adult life: a systematic review and meta-analysis. Pediatrics 131:e1240-e1263. https://doi.org/10.1542/peds.2012-2177

14. Strydom K, Van Niekerk E, Dhansay MA (2019) Factors affecting body composition in preterm infants: assessment techniques and nutritional interventions. Pediatr Neonatol 60:121-128. https://doi. org/10.1016/j.pedneo.2017.10.007

15. Holford N (2010) Dosing in children. Clin Pharmacol Ther 87:367370. https://doi.org/10.1038/clpt.2009.262

16. Ahmad I, Nemet D, Eliakim A, Koeppel R, Grochow D, Coussens M, Gallitto S, Rich J, Pontello A, Leu S, Cooper DM, Waffarn F (2010) Body composition and its components in preterm and term newborns: a cross-sectional, multimodal investigation. Am J Hum Biol 22:69-75. https://doi.org/10.1002/ajhb.20955

17. Morrison KM, Ramsingh L, Gunn E, Streiner D, Van Lieshout R, Boyle M, Gerstein H, Schmidt L, Saigal S (2016) Cardiometabolic health in adults born premature with extremely low birth weight. Pediatrics 138:e20160515. https://doi.org/10.1542/peds.2016-0515

18. Peralta-Carcelen M, Jackson DS, Goran MI, Royal SA, Mayo MS, Nelson KG (2000) Growth of adolescents who were born at extremely low birth weight without major disability. J Pediatr 136: 633-640. https://doi.org/10.1067/mpd.2000.104291

19. Saigal S, Stoskopf B, Streiner D, Paneth N, Pinelli J, Boyle M (2006) Growth trajectories of extremely low birth weight infants from birth to young adulthood: a longitudinal, population-based study. Pediatr Res 60:751-758. https://doi.org/10.1203/01.pdr. $0000246201.93662 .8 \mathrm{e}$
20. Hack M, Schluchter M, Margevicius S, Andreias L, Taylor HG, Cuttler L (2014) Trajectory and correlates of growth of extremelylow-birth-weight adolescents. Pediatr Res 75:358-366. https://doi. org/10.1038/pr.2013.209

21. Rodriguez-Soriano J, Aguirre M, Oliveros R, Vallo A (2005) Longterm renal follow-up of extremely low birth weight infants. Pediatr Nephrol 20:579-584. https://doi.org/10.1007/s00467-005-1828-6

22. Monset-Couchard M, de Bethmann O (2000) Catch-up growth in 166 small-for- gestational age premature infants weighing less than $1,000 \mathrm{~g}$ at birth. Neonatology 78:161-167. https://doi.org/10.1159/ 000014265

23. Group WMGRS (2006) WHO child growth standards: length/ height-for-age, weight-for-age, weight-for-length, weight-forheight and body mass index-for-age: methods and development. Geneva World Heal Organization 1-312

24. WHO (2007) WHO child growth standards: head circumferencefor-age, arm circumference-for-age, triceps skinfold-for-age and subscapular skinfold-for-age: methods and development. Geneva World Heal Organization

25. de Onis M (2007) Development of a WHO growth reference for school-aged children and adolescents. Bull World Health Organ 85: 660-667. https://doi.org/10.2471/BLT.07.043497

26. Moher D, Liberati A, Tetzlaff J, Altman DG (2009) Preferred reporting items for systematic reviews and meta-analyses: the PRISMA statement. PLoS Med 6:e1000097. https://doi.org/10. 1371/journal.pmed.1000097

27. Quality Assessment Tool for Observational Cohort and CrossSectional Studies. Retrieved from: https://www.nhlbi.nih.gov/ health-topics/study-quality-assessment-tools. Accessed 10 Dec 2019

28. Rodd C, Metzger DL, Sharma A, Cummings E, Chanoine JP, Lawrence S, Palmert M (2014) Extending World Health Organization weight-for-age reference curves to older children. BMC Pediatr 14:1-7. https://doi.org/10.1186/1471-2431-14-32

29. Matlab (1994) The Mathworks, Inc.

30. Raaijmakers A, Jacobs L, Rayyan M, van Tienoven TP, Ortibus E, Levtchenko E, Staessen JA, Allegaert K (2017) Catch-up growth in the first two years of life in extremely low birth weight (ELBW) infants is associated with lower body fat in young adolescence. PLoS One 12:e0173349. https://doi.org/10.1371/journal.pone. 0173349

31. Jordan IM, Robert A, Francart J, Sann L, Putet G (2005) Growth in extremely low birth weight infants up to three years. Neonatology 88:57-65. https://doi.org/10.1159/000084701

32. Saigal S, Stoskopf BL, Streiner DL, Burrows E (2001) Physical growth and current health status of infants who were of extremely low birth weight and controls at adolescence. Pediatrics 108:407415. https://doi.org/10.1542/peds.108.2.407

33. Starzec K, Klimek M, Grudzień A, Jagla M, Kwinta P (2016) Longitudinal assessment of renal size and function in extremely low birth weight children at 7 and 11 years of age. Pediatr Nephrol 31:2119-2126. https://doi.org/10.1007/s00467-016-34136

34. Atkinson SA, Steele S, Stoskoff B, Saigal S (2005) Program abstracts. Pediatr Res 58:1008-1139. https://doi.org/10.1203/ 00006450-200511000-00033

35. Crane JD, Yellin SA, Ong FJ, Singh NP, Konyer N, Noseworthy MD, Schmidt LA, Saigal S, Morrison KM (2016) ELBW survivors in early adulthood have higher hepatic, pancreatic and subcutaneous fat. Sci Rep 6:31560. https://doi.org/10.1038/srep31560

36. Doyle LW (2004) Extremely low birth weight and body size in early adulthood. Arch Dis Child 89:347-350. https://doi.org/10. 1136/adc.2002.025924

37. Hill AS, Nguyen H, Dickerson KL (2009) Catch-up growth for the extremely low birth weight infant. Pediatr Nurs 35:181-188 Retrieved from https://www.pediatricnursing.org 
38. Hirata T, Bosque E (1998) When they grow up: the growth of extremely low birth weight $(\leq 1000 \mathrm{gm})$ infants at adolescence. J Pediatr 132:1033-1035. https://doi.org/10.1016/S0022-3476(98) 70404-4

39. Kwinta P, Klimek M, Grudzień A, Piatkowska E, Kralisz A, Nitecka M, Profus K, Gasińska M, Pawlik D, Lauterbach R, Olechowski W, Drożdż D, Pietrzyk JJ (2012) Assessment of somatic development and body composition in the 7th year of life in children born as extremely low birth weight infants $(\leq 1000 \mathrm{~g})$; a multi-centre cross-sectional study of a cohort born between 2002 and 2004 in the Malopolska voivodship. Med Wieku Rozwoj 16: 81-88 Retrieved from http://www.medwiekurozwoj.pl. Accessed 10 Dec 2019

40. Lin Y-C, Lin Y-J, Lin C-H (2011) Growth and neurodevelopmental outcomes of extremely low birth weight infants: a single center's experience. Pediatr Neonatol 52:342-348. https://doi.org/10.1016/ j.pedneo.2011.08.008

41. Mól N, Zasada M, Klimek M, Kwinta P (2017) Somatic development and some indices of lipid metabolism in 11-year-old children born with extremely low birth weight $(\leq 1000 \mathrm{~g})$ (long-term cohort study). Dev Period Med 21:361-368 Retrieved from http://www. medwiekurozwoj.pl. Accessed 10 Dec 2019
42. Sices L, Wilson-Costello D, Minich N, Friedman H, Hack M (2007) Postdischarge growth failure among extremely low birth weight infants: correlates and consequences. Paediatr Child Health (Oxford) 12:22-28. https://doi.org/10.1093/pch/12.1.22

43. Powls A, Botting N, Cooke RWI, Pilling D, Marlow N (1996) Growth impairment in very low birthweight children at 12 years: correlation with perinatal and outcome variables. Arch Dis Child Fetal Neonatal Ed 75:F152-F157. https://doi.org/10.1136/fn.75.3. F152

44. Cooke RWI (2003) Growth impairment in the very preterm and cognitive and motor performance at 7 years. Arch Dis Child 88: 482-487. https://doi.org/10.1136/adc.88.6.482

45. Stathis SL, O'Callaghan M, Harvey J, Rogers Y (1999) Head circumference in ELBW babies is associated with learning difficulties and cognition but not ADHD in the school-aged child. Dev Med Child Neurol 41:S0012162299000833. https://doi.org/10.1017/ S0012162299000833

Publisher's note Springer Nature remains neutral with regard to jurisdictional claims in published maps and institutional affiliations. 